\title{
Application of the multi-curve reconstruction technology in seismic
}

\author{
inversion \\ WANG Yun-zeng \\ Hailaer Exploration and Development Headquarter of Daqing Oilfield Company, Hailaer 021000, \\ China
}

Keywords: well logging, standardization, multi-curve reconstruction technology, seismic inversion

\begin{abstract}
Well logs are usually used to constrained inversion during reservoir predicting. However the seismic inversion cannot be accurately guiding because of the logs affected by the various factors, which will affect the accuracy and reliability of prediction. Combined with the actual situation of Saertu areas, SP and RMN curve is fitted through multi-curve reconstruction technology. Remove the interference information, and remain the valid information of SP curve against the way that errors generate. Suppress the effects of high lithological differences and random interference to their baseline by standardization, normalization, linear filtering method and then fit SP and RMN curves. Proposed high-resolution, high-fidelity curve reconstruction, seismic inversion guidance sand prediction, and achieved good results [1]. This indicates that the method is a simple, practical and effective curve reconstruction techniques can be promoted in practical applications.
\end{abstract}

\section{Introduction}

Logging constrained inversion of seismic reservoir is a well logging, seismic, geological formations and other data of the wave impedance (or speed) inversion technique. With the inversion continuous development of technology, there are now a variety of wave impedance inversion method, the correct application of these methods is the key to describe the reservoir, while effectively utilizing the raw data is crucial inversion [2]. Therefore, analysis and application logging data is particularly important seismic inversion.

With the development of in-depth exploration, the requirements of reservoir prediction accuracy are high, so the requirements for sonic log data used in the seismic inversion is also rising. Only the results of the inversion can effectively reflect the speed or wave impedance difference between the reservoir and the surrounding rocks, reservoir can be described correctly [3]. However, the differences between the reservoir and the surrounding rock are small because the reservoir subsurface has a strong reservoir space complexity, controlling factors and more features. In addition, in many cases, the well logs ,which are used for seismic and geologic horizon calibration and wave impedance inversion, are enable to reflect the changes of lithology [4] due to the impact of pollution wellbore formation and the degree of compaction logging conditions and other factors. It is particularly obvious when subsurface horizons are thin and inter bedded.

\section{Multi-curve reconstruction}

In this article, SP curve and RMN curve is selected. Firstly, the correspondence between the two curves and lithology are analyzed to find out the reasons for the distortion effects then their active ingredients are retained. Finally, the curve is fitted by a suitable method. The process includes baseline drift, standardization, linear filtering, curve fitting. 
SP curve processing. The baseline of SP will deflect when influenced by geological factors and causes of water exploitation [5]. So its baseline must be corrected appropriately, which is shown in Fig.1 and Fig.2.

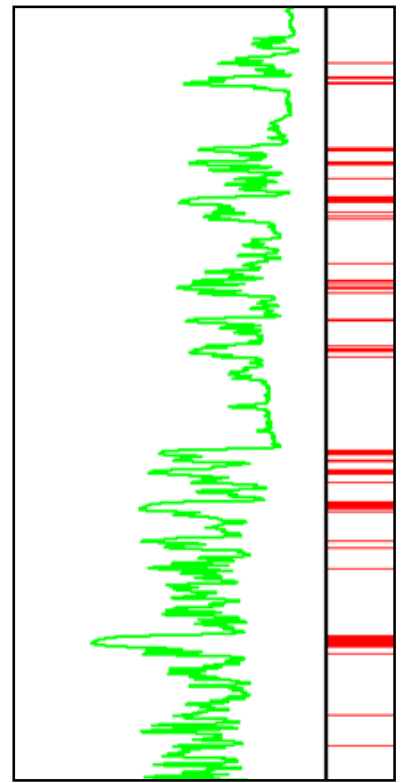

Fig.1 SP curve before drift

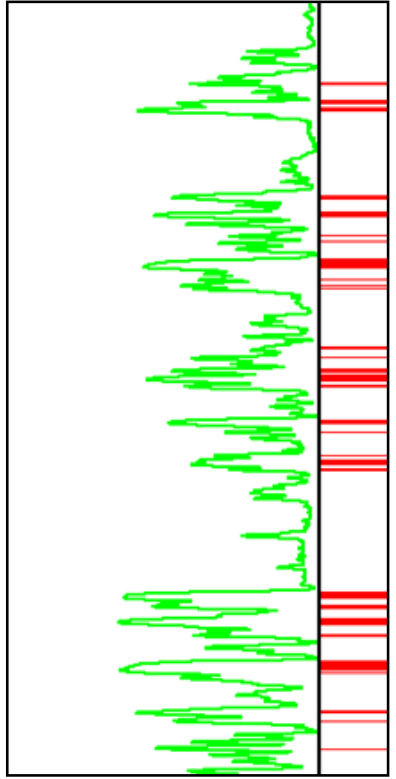

Fig.2 SP curve after drift

The value of SP curve can vary greatly due to the geological conditions and water conditions, which brings great difficulties to the next work. So it seems very essential to correct the value of SP curve which is shown in Fig.3 and Fig.4.

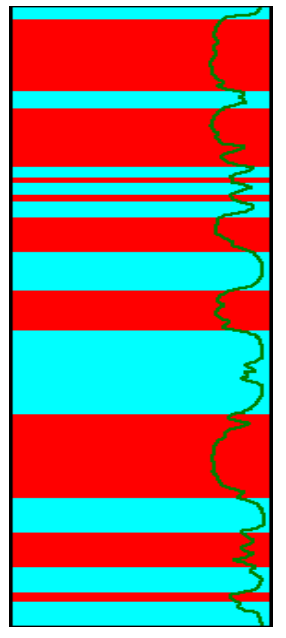

Fig.3 SP before normalization

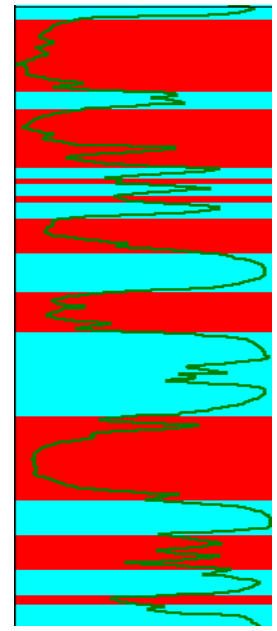

Fig.4 SP after normalization

RMN curve processing. The rive sands of Saertu during thin horizons develop well. Thin horizons that contain calcium are often found, which seem knife in the curve and will bring difficult to next work. It may mislead the result of seismic inversion. For this problem, how to deal with this abnormal values become very import and its effect will reflect on the result of inversion directly. Naturally, an appropriate mean should be taken--linear filtering is proposed, which sets a range of normal value and then fills proper values in abnormal range. In this way the negative effect of calcium horizons is effectively repressed.

The structure of this area varies quickly and has huge difference, which result in a large difference curve value of the RMN curve. For this problem, the approach of trend surface standardization is chosen because this method can solve this problem effectively [6].

A basic model is established to study by trend surface fitting formula several times duality equation like this: 


$$
Z^{\prime}=B_{1}+B_{2} X+B_{3} Y+B_{4} X^{2}+B_{5} X Y+B_{6} Y^{2}+\ldots+e
$$

In this equations, $e$ is the random deviation; $B_{i}(i=1,2, \ldots, p)$ is undetermined coefficients of the polynomial $Z^{\prime} ; \quad X, Y$ is the location coordinates.

Due to the measured point number is limited, fitting measuring point is limited, and it can be changed the fitting polynomial to:

$$
Z=B_{1}+B_{2} Y+B_{3} Y+B_{4} X^{2}+B_{5} X Y+B_{6} Y^{2}+\ldots
$$

Square and random deviations for optimal estimation to determine the coefficients of the polynomial, as follows:

$$
f=e=\left(Z^{\prime}-Z\right)=Z^{\prime}-\left(B_{1}+B_{2} Y+B_{3} Y+B_{4} X^{2}+B_{5} X Y+B_{6} Y^{2}+\ldots\right)=f_{\min }
$$

Making it zero-order partial derivatives:

$$
\frac{\partial f}{\partial B_{i}}=0(i=1,2, \ldots, n)
$$

Solving the equations, the coefficients $B_{1}, B_{2}, \ldots$ can be obtained.

In this process, the geological information is effectively joined to make the standardization more meaningful through improving parameters.

\section{Seismic inversion}

High quality curve ensure the accuracy and reliability of the well logs constraint seismic inversion. The reconstructed curve is obtained on the basis of several tests in this area. The reconstructed curve promises these:

It has high resolution because the RMN curve has high resolution;

It has high stability.

The results contrast. It is shown in Fig.5 and Fig.6.

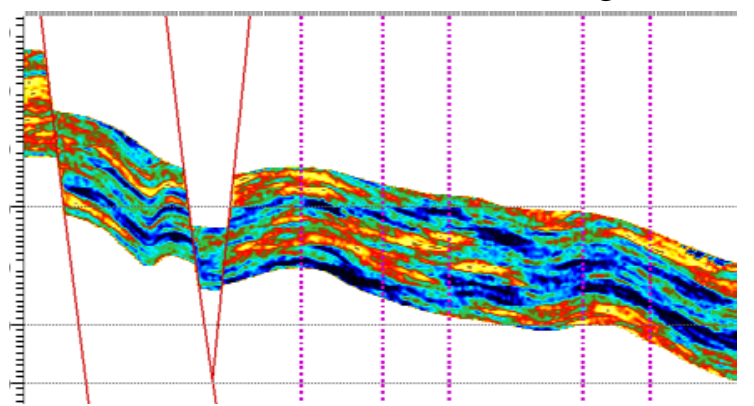

Fig. 5 Impedance inversion

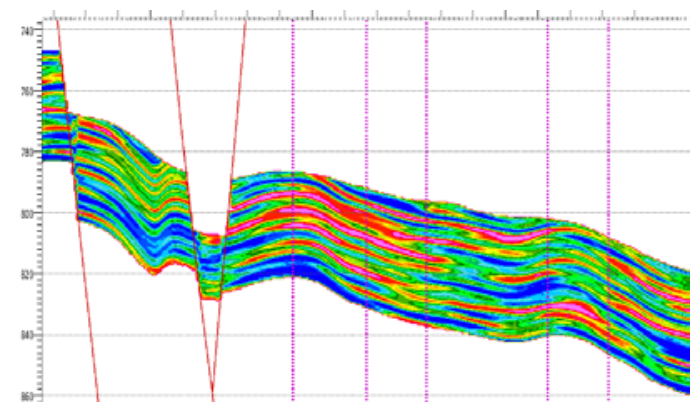

Fig.6 reconstructed curve inversion

Map of Sandstone thickness. The high resolution and accuracy of multi-curve inversion is shown in Fig.7, the map of sand thickness, which are extracted from the results of inversion data.

Error statistics: $<2 \mathrm{~m} 93 \%$. 

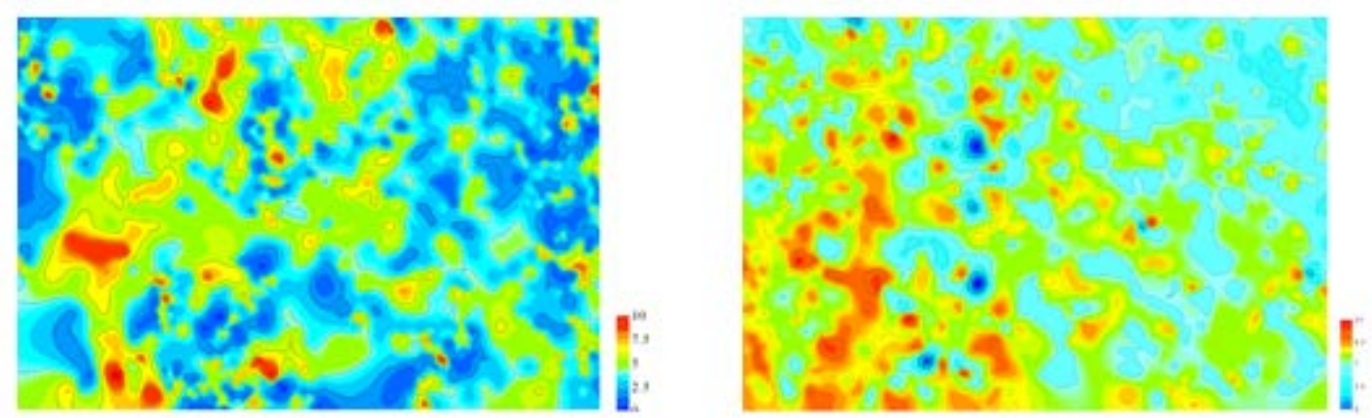

Fig.7 map of multi-curve inversion

\section{Conclusion}

For the conventional problem-low resolution and low credibility, high quality multi- curve reconstruction is proposed through analyzing the sources of noise, improving coefficient, suppressing the noise by linear means. The result of the prediction of distribution of sands proves that the high quality multi- curve reconstruction seismic inversion can solve low resolution and low credibility with little error.

\section{References}

[1] Xu Jianjun, Xu Yan-chao, Yan, Li-me,et.al. Research on the method of optimal PMU placement. International Journal of Online Engineering,v9, S7, p24-29, 2013

[2] Xu Jian-Jun, Y. Y. Zi., Numerical Modeling for Enhancement of Oil Recovery via Direct Current. International Journal of Applied Mathematics and Statistics，2013，43 (13) : 318-326

[3] Longchao, Zhu Jianjun, Xu; Limei, Yan. Research on congestion elimination method of circuit overload and transmission congestion in the internet of things. Multimedia Tools and Applications, p 1-20, June 27, 2016

[4] Yan Limei, Zhu Yusong, Xu Jianjun,et.al. Transmission Lines Modeling Method Based on Fractional Order Calculus Theory. TRANSACTIONS OF CHINA ELECTROTECHNICAL SOCIETY, 2014 ,Vol.29,No. 9:260-268 (In Chinese)

[5] YAN Li-mei, CUI Jia, XU Jian-jun,et.al. Power system state estimation of quadrature Kalman filter based on PMU/SCADA measurements. Electric Machines and Control. 2014, Vol.18 No.6,: 78-84. (In Chinese)

[6] Xu J.J., Gai D., Yan L.M. A NEW FAULT IDENTIFICATION AND DIAGNOSIS ON PUMP VALVES OF MEDICAL RECIPROCATING PUMPS. Basic \& Clinical Pharmacology \& Toxicology, 2016,118 (Suppl. 1), 38-38 